\title{
Smartphone Crowdsourcing and Data Sharing Towards Advancing User Experience and Mobile Services
}

\author{
https://doi.org/10.3991/ijim.v14i03.11815 \\ Aristea Kontogianni $\left.{ }^{\varpi}\right)$, Efthimios Alepis \\ University of Piraeus, Piraeus, Greece \\ akontogianni@unipi.gr
}

\begin{abstract}
Recent advances in IT have offered people the opportunity to have powerful ultramobile devices in their pockets incorporating a plethora of capabilities which, in most cases, require Internet connectivity. This constant need for Internet access though, especially when a wireless hotspot is unavailable requires connection via network data. The fixed cellular data packages offered by carriers usually do not come for free and to a large extend cannot be adapted to the dynamic needs of users who sometimes need more than they own, while in other cases under-use their network data. This issue, in combination with the fact that signal quality in many areas is not even fair for one or more carriers has led to the development of a novel android application that aims at allowing users in general and tourists to foresee bad signal coverage at places of their interest, as well as to share their cellular data via mobile tethering.
\end{abstract}

Keywords - Smartphone application, crowdsourcing, smart-tourism, mobile signal coverage, sensors, social application, data sharing.

\section{Introduction}

Mobile devices become more and more popular all over the globe, as they offer time and space independence [1,27], forming a tight" bond" between them and the users as they always tend to be close by [2]. More specifically, statistics indicate that $78 \%$ of users are using their smartphones more than they did a year ago, while the corresponding growth for laptops/desktops is limited to $42 \%$ [2]. This increasing popularity of the aforementioned devices along with the fact that modern mobile devices are real competitors of desktop computer systems with regard to hardware and software [3], allow users to use them for a plethora of functionalities, ranging from downloading and using data, to socializing and accessing emails. In particular, user interaction with mobile devices has been recently counted in terms of tens per hour [2].

With the above being said, a demand has arisen to access Internet-based services via mobile terminals [4] with statistics showing that the $52.2 \%$ of website traffic worldwide in 2018 was generated through smartphones [5]. Nonetheless, what is even more interesting is that smartphone users, instead of browsing the internet, prefer to 
utilize apps when using their mobile phones, while the majority of these apps require Internet connectivity. Particularly, TACYT ${ }^{1}$, a tool that downloads on a daily basis all applications from Google Play as well as other available markets and analyses them, indicates that more than $90 \%$ of android applications publicly available require Internet access [6]. These findings come at no surprise since during our recent past user data have been considered as the "new oil", not only providing individualized help and adaptation to users, but also great economic profits for companies around the world [7].

Regardless of the aforementioned reasons, we are witnessing an era where smartphones and mobile devices in general have become some kind of "wearable", following users everywhere and at any time and allowing them to connect with others, perform a plethora of actions, access Internet based services, point their location [28] etc. As a result, they are also considered as powerful tools while traveling [29], where users tend to need as much "guidance" as possible. To that end, smart tourism applications draw increasing attention, as the aforementioned need does not pass unnoticed, with more and more destinations trying to transform themselves to Smart Locations, applications that require Internet access $[8,9]$.

In the cases Wifi connection is of poor quality or even unavailable, users rely on accessing the Internet via operator networks. This option requires consuming cellular data that, in many cases do not come for free. That being the case, along with the fact that having sufficient phone signal is necessary so as to access the Internet and take advantage of all capabilities that mobile devices offer. In view of the above, the basic aim of this paper is the creation of a novel android application that shall pose a solution to all issues mentioned above.

More specifically, in this work we present a two-year study that resulted in the creation of a crowdsourcing android application [10] that visualizes signal coverage and signal quality on a map interface. At the same time, it enables users to locate other users around them that are willing to offer Internet connection by turning their mobile devices to an instant HotSpot access point. More specifically, the developed application offers its' users the ability to view signal quality samples from all over the globe that have been gathered for an evaluation period of 24 months, in 17 countries around the world. In particular, the experimental results that derive from participatory sensing [6] via the android application indicate that signal coverage is not the expected/advertised one and surely not the same for all carriers at a specific place. As a test bed for our signal evaluation study, from the 13 participating countries we have chosen the one that offered the largest amount of data in our database, namely Greece. In fact, in countries like Greece that highly engage in the tourism industry can cause a plethora of issues, since, evidently, signal coverage services in many touristic destinations is ranging from "bad" to even "zero". The developed app targets at offering a solution to the Internet connection issues users may face, allowing them to both predict a possible connectivity problem in a specific location, as well as resolve it via connection to other users with stronger signal connections via mobile tethering.

\footnotetext{
${ }^{1}$ https://www.elevenpaths.com/technology/tacyt/index.html
} 
The remainder for the paper is structured as follows: Section 2 contains related works. In Section 3 we set the problem the developed application aims to resolve. In Section 4 we present the architecture of the system while Section 5 consists of an overview of the application. Finally, in Section 6 conclusions and future work are discussed.

\section{Work}

We are witnessing an era where mobile devices, like smartphones and tablets, with numerous integrated sensors enable the low-cost recording, storage and transmission of real time data [11] regarding the environment of their owners. With that being said, the idea of transforming mobile devices into sensors grew to be quite appealing, which unavoidably gave birth to the concepts of participatory and mobile crowd sensing $[12,13]$. Indeed, due to the enormous number of devices, sensing through smartphones has huge potential [14]. At the same time, mobile terminals can serve as a crowd, so as to lead to the creation of collective intelligence in order to resolve an issue. In view of the above, multiple crowdsourcing - crowdsensing applications have been developed aiming to suggest solutions to a plethora of problems.

Sheng et al. [15] introduce the Sensing as a Service concept and present a crowdsourcing system that consists of a number of Sensing Servers that are used to hand over requests made by a system user to a number of mobile devices placed in a specific location. The aforementioned devices perform the requested sensing task and deliver the produced data to the Sensing Servers. The gathered information is stored in a database and transferred back to the system user who performed the initial request.

Moreover, authors in [16] also harness the power of the crowd so as to obtain signal coverage data combined with location ones in order to save energy for cellular data communications. The motivation of this research is the fact that when the quality of signal is poor more energy is consumed when using cellular data. In view of that, they suggest a crowdsourcing approach where signal strength traces collected by a plethora of smartphones can be exploited, once they are filtered so as to predict the signal quality in a location. By doing so, optimization of mobile devices energy efficiency is realized by prefetching content in places where signal coverage is sufficient while postponing packets in those that is not.

Another both crowdsourcing and crowdsensing approach, presented in [17], tries to measure the network quality, nevertheless the authors aim to improve the services offered by network providers. In particular, their goal is to offer to network providers a single indicator regarding the network quality of a base station or a cell, by aggregating measurements obtained from a crowd of users in a specific location, so as to assist them in locating signal issues and take action to resolve them. Moreover, users' usage patterns can be obtained and used for future planning. A main difference between this related work and ours, is that a main priority for us is to benefit users and offer them insights about signal coverage in the locations of their choosing and consequently provide them with alternative solutions via data sharing. 
Crowdsourcing applications seem to attract increasing attention in a plethora of sectors, as discussed in [18] [19]. The system presented in [20], attempts to resolve the 3-power exhaustion issue in mobile devices via a system that allows users that wish to share their battery to get in contact with each other. An attempt to propose a solution to the non-dynamic plans issue offered by carriers is made in [21]. Authors here also locate the problem of waste or overuse of cellular data in combination with that of the low Internet quality and aim at resolving it with a system that allows mobile devices to cooperate so as to download data from the Internet. The aforementioned system is based on unfamiliar users that share network data resources in order to reduce cellular data usage of a single user while increasing the download speed.

In this research we attempt to combine the concepts of crowdsourcing applications with participatory sensing so as to propose a system that allows users to share their cellular data via tethering, while considering the signal coverage in an area of interest and actively contribute to the data harness via mobile sensing. While the are some papers in the related scientific literature that aim at gathering signal samples through users mobile devices, after a thorough investigation in the related scientific literature we did not locate a system that combines the signal coverage information with a crowdsourcing application that allows users to both act as data donors by sharing their cellular data and connect to the Internet via other users' network data.

\section{$3 \quad$ Problem Setting}

Mobile phones have established their presence in our daily lives, with the most recent analytics indicating that, almost $80 \%$ of mobile users use their smartphones the current year more than they did the previous one [2] as also mentioned in a previous section. At the same time, mobile data traffic has grown 18 -fold over the past 5 years, with the global mobile data reaching 7.2 exabytes per month at the end of 2016 [4]. That being the case, it comes as no surprise that being able to access the Internet through mobile devices anywhere at any time has become a pressing issue both for users and also for the involved companies. Nevertheless, accessing the Internet through smartphones, may be in some cases challenging.

Evidently, there is a plethora of times during peoples' daily activities where WiFi connection is either slow or even unavailable, making the consumption of mobile network data a one-way solution. Being constantly connected to social media channels, streaming videos, accessing emails, as well as performing other online activities, though, requires huge chunks of mobile data that the majority of carriers, if not all, do not freely offer. To this end, there may be cases where users need to purchase more data than their program offers, while, there may also be cases where users do not use the whole amount of data they own, resulting in a waste of data. The aforementioned facts lead to the conclusion that the fixed network data packages offered by carriers, run counter to the dynamic cellular data needs of users.

Another critical issue that highly affects tourists is that while traveling to a different country the cost for using cellular data may be extremely high. In that case and given the fact that $\mathrm{Wi}-\mathrm{Fi}$ is not available in every location, tourists may find them- 
selves unable to use the offered services, which require Internet connection that in many cases may be extremely important [22].

Furthermore, there is a plethora of cases where users do have sufficient mobile data to use but the quality of the signal in a particular location does not allow it. Based on experimental results that derive from data gathered via participatory sensing, an experiment that goes on for a period of two years, we came to the conclusion that the aforementioned issue actually is more pressing than it appears to be by carriers, which tend to advertise their signal coverage and stands a major challenge to be objectively presented and dealt with.

Table 1: Signal Strength (dBm) Value Explanation

\begin{tabular}{|c|l|l|l|}
\hline Signal strength(dBm) & \multicolumn{1}{|c|}{ Map color } & \multicolumn{1}{|c|}{ Quality } & \multicolumn{1}{c|}{ Meaning } \\
\hline$>-60 \mathrm{dBm}$ & Green & Excellent & Device is close to a cell tower \\
\hline-60 to $-90 \mathrm{dBm}$ & Light Green & Good & Decent coverage \\
\hline-91 to $-99 \mathrm{dBm}$ & Orange & Fair & Coverage may be slow \\
\hline 100 to $110 \mathrm{dBm}$ & Red & Poor & Probability of connectivity issues \\
\hline$\leq-110 \mathrm{dBm}$ & Black & Zero & A call is impossible to be performed/ completed \\
\hline
\end{tabular}

More specifically, we have developed and distributed, over the corresponding marketplace, an android application that aims at collecting signal quality samples in combination with location points, via crowdsourcing. The app is based on participatory sensing, where users' mobile devices serve as a sensor crowd so as to measure the signal quality in a particular location along with some other information regarding the device and the signal coverage and thereafter store them in real time on the cloud. After conducting an experimental study via this app for 24 months, we may safely claim that there are specific areas in the countryside, the capital and/or at specific timeframes (e.g. in concerts) where signal quality is poor for one or more carriers. At the same time, other carriers may not suffer from such issues at the given location/time.

Particularly, up to the time of writing about 200 registered users have contributed to the data harness with samples originating from 17 countries all over the globe. A quite extended database of 126.497 samples is produced while approximately 12.600 $\mathrm{km}$ of land distance have been covered. The experimental results signify severe issues regarding the quality of services provided as well as the signal coverage. Indicatively, as presented in Figure 3.1 only the $2.1 \%$ of the samples collected illustrate excellent signal quality while approximately $33.9 \%$ of them indicate good signal quality. What is quite alarming is that almost $25.2 \%$ of the samples recorded display poor signal coverage and approximately $13.9 \%$ of them zero signal quality, which actually means that users are not able to perform a call, let alone to connect to the Internet via their cellular data. The aforementioned results derive from Signal Strength values (measured in $\mathrm{dBm}$ ) which are presented in Table 1 [23]. With that being said, a demanding need arises, namely the ability to inform users regarding the signal coverage in particular locations, as well as offering them an alternative way to connect to the Internet, when Wi-Fi is unavailable or poor. 
An observation deriving from the aforementioned experiment is that there are areas where particular carriers suffer from coverage issues while others don't. What is actually a major finding is that in a country like Greece where the size of the tourism industry is huge, the experimental results for rural areas like islands reveal that the signal quality for the most carriers is zero while other carriers offer good, even excellent signal. This fact might be known to locals but not to tourists who usually face an unpleasant surprise. As an example, we present the following use case where signal samples are collected in the same rural locations for two different carriers. The islands presented attract loads of tourists during summer vacations, with a wide number of visitors following the same routes and locations presented in Figures 3.2 each day. The experimental results undoubtedly present that while one carrier has mostly good or even excellent signal quality, the other one has mostly zero signal coverage. That being the case, there is an arising need for the users to be able to be informed regarding such issues, as well as being offered the opportunity to access the Internet via alternatives, such as the cellular data of another mobile device that does not face the same problems.

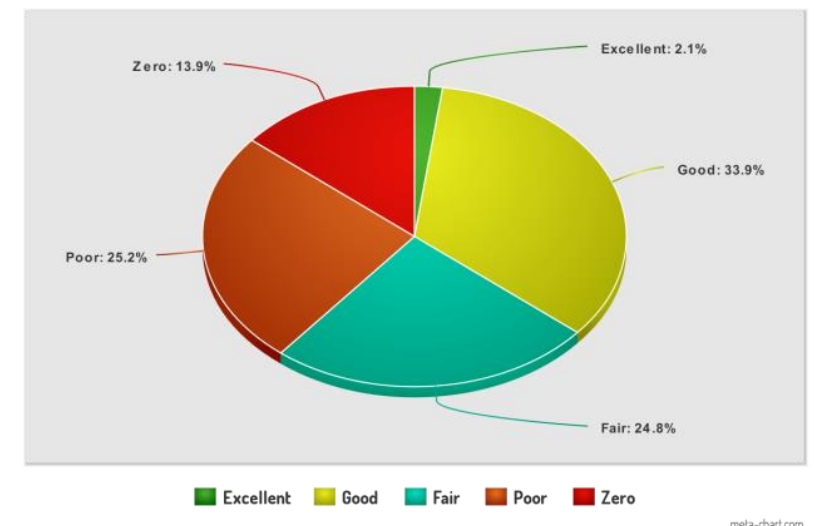

Fig. 1. Signal Quality Results

Moreover, with tourism considered one of the biggest sectors worldwide [24] and a crucial part for the economic growth of countries, it seems quite important to be able to offer tourists a pleasant experience during their travels. With that being said and given the fact that an increasing interest in creating Smart Destinations has arisen [25] with platforms enabling the creation of real-time tourist experiences [26], the need to offer smart tourist services via smart tourism applications, renders Internet connection necessary. After a thorough analysis of the aforementioned dataset, an "unpleasant" ascertainment came to light regarding a country that is highly involved in tourism, namely Greece, where urban areas such as islands, villages, beaches as well as a high percentage of highways and roads suffer from poor signal quality and, in some cases, even total absence of signal. An example of the signal coverage to a large part of Greece that includes highways, touristic areas and islands is presented in Figure 3.3. To that end, it seems quite important to be able to offer insight to tourists regarding 
signal issues that may arise to locations they intend to visit as well as a way to resolve them, guaranteeing a more pleasant experience, a way to avoid disturbing or even dangerous situations and an unlimited access to smart tourism applications. At the same time, it is the authors' strong belief that through tourist feedback, authorities may be informed about signal quality issues in areas of high tourist interest.

Last but not least, there is the vital issue about energy consumption in smart mobile devices when using cellular data, in which signal strength plays a crucial role [16].To that end, it seems quite important to be able to predict signal quality in locations of interest, based on signal traces harnessed by other users, as well as having the ability to connect to the Internet utilizing another users mobile device as HotSpot. The aforementioned features are both offered via the proposed application which allows its' users to optimize energy consumption by avoiding connections to the Internet via cellular data when signal quality is poor and offering them an alternative.

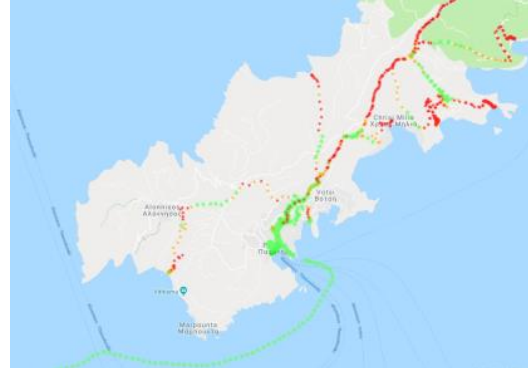

(a) Signal coverage for carrier $\mathrm{A}$

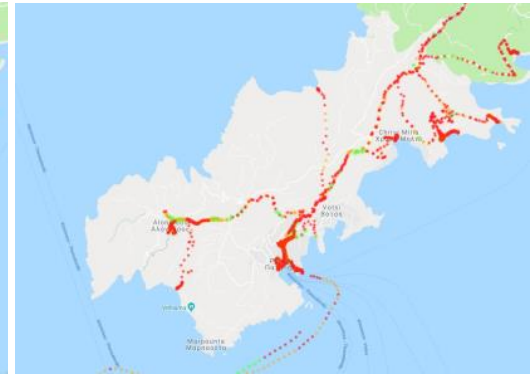

(b) Signal coverage for carrier

Fig. 2. Signal coverage for two carriers in the same location

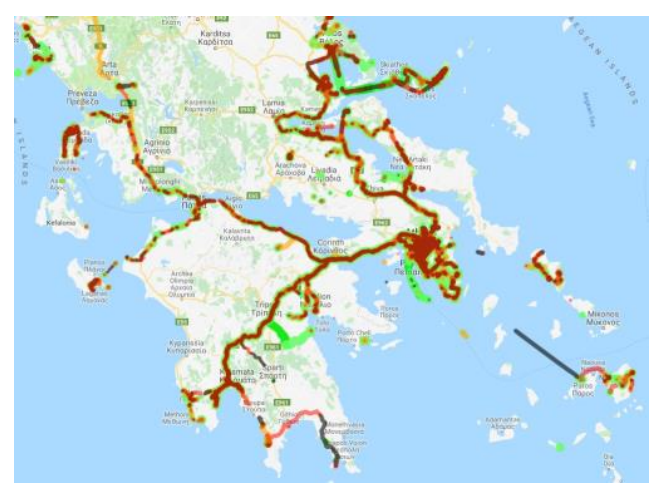

Fig. 3. Signal Heatmap Instance Example covering the major cities and highways of Geece 


\begin{tabular}{l} 
Excellent Signal \\
\hline Good Signal \\
\hline Fair Signal \\
\hline Poor Signal \\
\hline Zero Signal \\
\hline
\end{tabular}

Fig. 4. Signal quality representation

\section{System Architecture}

Within the scope of this research we have designed a mobile application towards a solution to the aforementioned issues. In particular, we present a system that supports interaction between users that wish to share their data, along with a valid feedback regarding signal coverage in a desired location. To this end, we have developed a social network mobile application that allows users to easily identify the signal quality in a location, visualize nearby users that are able to share their cellular data in the aforementioned location and record the frequency of data sharing required by tourists in particular places of tourist interest so as to offer a better insight about how poor signal coverage may affect the tourism industry.

\subsection{The application}

The application presented in this paper aims at exploiting the signal coverage dataset produced by our experiment, allowing users to have an insight regarding the quality of the signal in a desired location, along with the ability to share their abundance of cellular data. At the same time, for every byte shared a number of virtual coins is credited to their account for future usage.

More specifically, the sharing application module targets individuals that wish to share their mobile network data with others via tethering. The aforementioned system is a native android social network type application that initially requires users to create a profile so as to be credited with a number of virtual coins (e.g. 200 coins). These coins are analogous to the number of Mbytes of data users consume when connected to the internet via tethering, while using the application.

The app's main UIs, corresponding to the app's basic operational functionalities, allow users to view their profile, their list of contacts along with their sent/received pending contact requests as well as a two map interfaces. The first one is used to locate users within a certain radius and present their signal strength. Available users appear with different color indicators on the map based on the category they belong to: existing contacts, unknown users, friendship request made by/to the user where confirmation is still pending. By clicking on the aforementioned indicators, the profile of the possible "data donor" is loaded, where details like his/her rating, current signal strength and virtual coins are presented along with the option to request via notifica- 
tion data donation, if a user is an existing contact, or to send a friend request in other cases.

The process for requesting data from another user via the application and connecting to a HotSpot is described in the following use case:

- User sends a request for Internet connection.

- The potential data donor receives a notification regarding the request.

- When the request is accepted, the application generates two random string of ten and fifteen characters that serves as the HotSpot name and password additionally in the device that shall share network data.

- In the same device, the application enables tethering with the credentials mentioned above.

- A notification that contains the aforementioned credentials is sent automatically to the user that requested the Internet access, which informs him/her that access is granted.

- By clicking on the connect option in the notification, the application locates the Hotspot in question via the name received along with the notification and connects via the also received password.

- For each byte used, a number of virtual coins are credited to the data donor. At the same time, this number is subtracted from the virtual coins of the client user. This actually serves as an incentive mechanism so as to make sure that all users will, at some point, serve as" data donors" in order to be able to "pay" future connections.

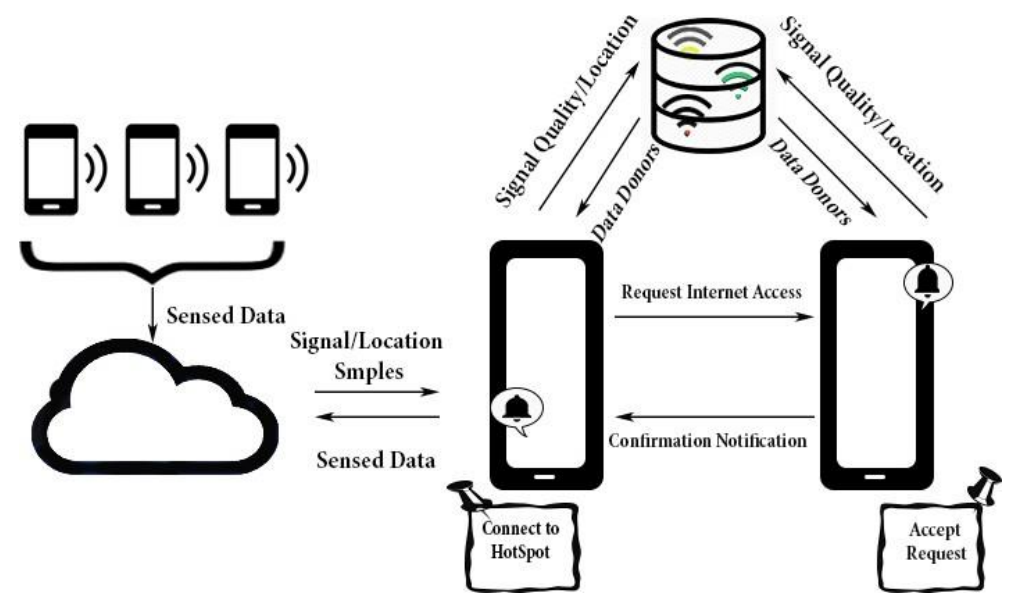

Fig. 5. : System Structure

The second map interface is used to load all the signal samples. These signal samples derive from the app's crowdsourcing module which collects via participatory sensing information regarding signal quality combined with the longitude and latitude of a mobile terminal at a specific time. The sensed data is sent on real time mode to a Firebase database, a NoSQL cloud database, when Internet access is available. Firebases' off-line database feature allows us to store signal coverage data even in cases 
where Internet connection is not possible, as the aforementioned information are synced back to the cloud infrastructure once network connection is re-established.

The overall goal of this harness is to collect as many information regarding various aspects of signal coverage and quality as possible for a plethora of carriers and locations, so as to be able to provide some valid feedback which will eventually benefit mobile users, carriers and authorities. All data collected via this application are stored on the cloud and displayed on a Google Maps interface after being processed. Table 1 presented above, indicates how Signal Strength values (measured in $\mathrm{dBm}$ ) correspond to different colors on the heatmap based on the actual quality of a signal sample [23]. It is important mentioning that user's data are not connected to their personal information, they are totally anonymized.

The app's Map interface allows users to view the signal quality in every location they wish, if the additional information is available in the database so as to be prepared for potential connectivity issues. The corresponding data is analyzed and categorized depending on both the signal strength and the carrier. To this end, users have the ability to filter the presented data in order to see the signal quality only for the carriers they wish. Moreover, the data points are illustrated with a different color, depending on the quality which is measured in $\mathrm{dBm}$, as presented in Table 1. Another feature available in this map interface, allows users to locate potential "data donors" at a different location than theirs, by simply selecting a place on the map. With that being said, an additional usability is introduced that not only enables to foresee signal coverage issues but also requires to locate possible solutions before visiting a specific location

Aiming to offer the opportunity to users to communicate more freely and potentially arrange a data exchange, another feature is incorporated allowing instant messaging between users, for communication purposes. This direct communication, in combination with the aforementioned features, allows users to view the signal coverage in a location they plan to visit, load users that are willing to share data at that area, communicate with them in order to check their availability and even request/receive the acceptance notification required in order to connect to another users hotspot before visiting the location in question.

Furthermore, so as to overcome a major restriction that requires Internet access in order to locate users and request connection via Tethering, a complementary feature is incorporated allowing connection via Bluetooth. As is widely known, Bluetooth is used in order to send and receive data from other Bluetooth enabled devices. The distance at which the connection is possible can reach up to 30 meters with the precondition that the devices in question are in direct line of sight of each other while inside buildings it is reduced to 10-20 meters [27].

As mentioned previously, the initial signal quality dataset has been created by users via the android application, where there was no tangible motivation other than contributing to an experiment. In the proposed system users will also anonymously contribute to the signal data harness, so as to enhance the signal coverage database and be able to improve the offered services. Moreover, an extra option for users that visit a location as tourists is introduced, so as to more clearly identify signal coverage issues in areas of high tourist interest. 


\section{Application Overview}

In this section the proposed application is presented. More specifically, we describe the functionality of some basic UI of the system, along with some technical specifications. The bottom navigation bar of the proposed system contains four icons that lead to:

- Users Profile: Users can view and alter their personal information as well as monitor their available virtual coins

- A Map interface that loads potential data donors around a user

- A Map interface where:

- Signal coverage Heatmap can be loaded

- Data Donors in an alternative location can be loaded

- Users Contacts lists: Three available lists for received/pending contact requests and existing contacts

Once a user firstly accessed the application, she/he is automatically transferred to the map interface, which is integrated via the Google Maps Android API. There, by selecting the human icon pinned at the left corner of the UI, all "potential for data tethering" users within a radius (the default is set to five hundred meters) are displayed in the UI, as illustrated in Figure 5.1. Potential "data donors" are presented on this interface with a different icon, depending on two different properties they have:

- Their signal strength: more or less colored bars in the icon displayed that indicate if the signal is excellent, good, fair or poor based on Table 1

- If they are existing contact (green color), sent/received pending contact request (blue/orange color) or an unknown user (red color).

By selecting a marker on the aforementioned interface and clicking on the user name that corresponds to the marker in question, the "data donors" profile is loaded. In this UI, users' personal information are presented, along with other options like rating the user, deleting/adding him/her from/to the contacts list, or request data donation.

The Map interface that integrates the signal quality dataset is presented in Figure 5.2a. By selecting the signal icon in the top left corner of the screen, a Heatmap that contains the signal quality data is loaded, with different colors indicating the quality, based on Table 1. In the same Interface, users may locate in a radius in a particular location other than their current one, individuals available for tethering, which are presented in the screen with the same icons as the ones described above.

The Settings UI is illustrated in Figure 5.2b. This interface consists of options for the users, such as the ability to alter the view of the map, change user credentials, as well as an option so as to determine the data collection rate, namely "driving", "walking", or "standing". Moreover, there is also an option for tourists to select, so as to label the data samples sent to Firebase, in order to create, if possible, a clearer picture regarding areas of high touristic interest. 


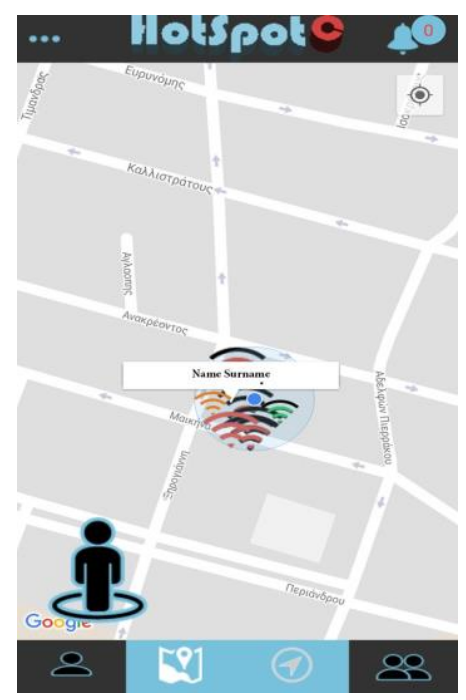

Fig. 6. : User Map Interface

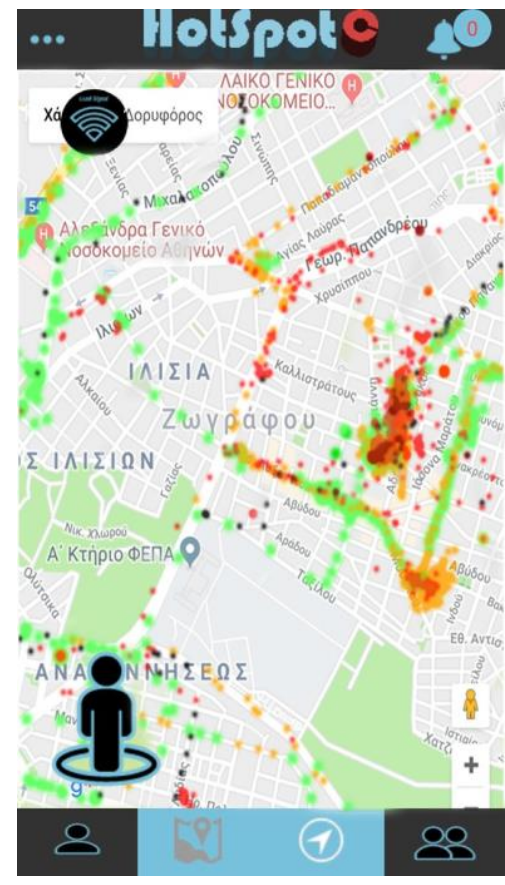

(a) Signal Map Interface

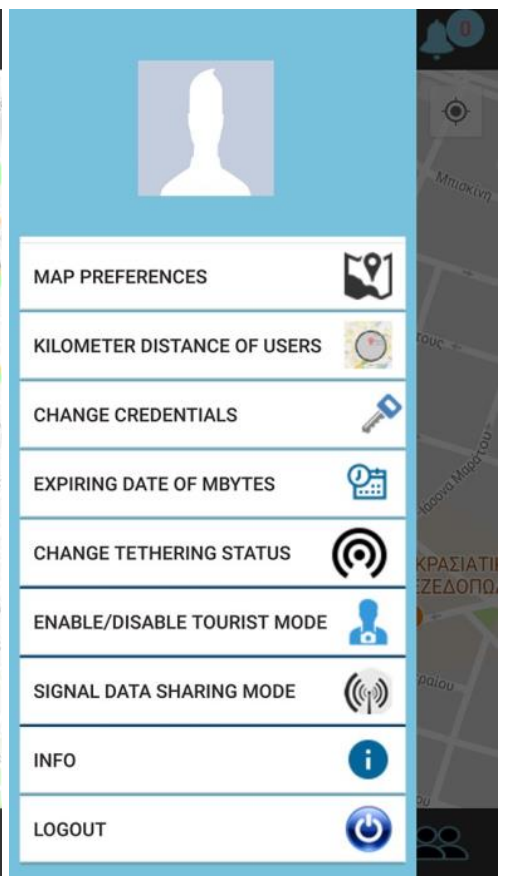

(b) Settings Interface

Fig. 7. : App User Interface 


\section{Discussion and Conclusion}

The presented research study aimed at presenting both the results and also the underlying innovative application that deals with resolving issues of the users' constant need of Internet access via mobile devices and unawareness of signal quality in locations of interest. Our system allows users to be a part of a social network where individuals wish to share their cellular data via mobile tethering. At the same time, they have access to a dynamically created and updated map in which user-contributed signal measurements are displayed, allowing them to foresee signal issues that may arise and locate possible solutions, as for example other users that shall be willing to grant them internet access via the application, or check if a mobile carrier can guarantee at least fair signal access.

Our experimental results indicate that there are a plethora of areas where signal quality is poor for one or more carriers while at the same time there is a fair number of problems that this system could offer solutions to, making it safe for us to claim that there is an actual need both for the presented application and also for the information that it offers to individuals. As an incentive mechanism for sharing cellular data, we have introduced a virtual coin's system that assures that all participants act also as "data donors". While using the application users contribute to the signal data harness, which is vital so as to enhance the already existing database and offer even better assistance to users.

It is in our future plans to proceed to a further and more extended evaluation of the presented application so as to actually define if such an idea would be widely accepted by users and tourists, by receiving actual feedback from users. At the same time, the success of this application would lead to the enlargement of our signal database which can be further exploited in a wide range of applications concerning customers, carriers, regulators and third-party companies.

\section{$7 \quad$ References}

[1] M. Virvou, E. Alepis, Mobile educational features in authoring tools for personalized tutoring, Computers \& Education 44 (1) (2005), 53 - 68 https://doi.org/10.1016/j. compedu.2003.12.020

[2] Generation $\mathrm{z}$ : Increase in device usage compared to a year ago, https://www.commscope.com/ Insights/chart-details/ increase-in-using-device-comparedto-year-ago/, accessed: 2018-06-04.

[3] A. Lazouski, F. Martinelli, P. Mori, A. Saracino, Stateful data usage control for android mobile devices, Int. J. Inf. Sec. 16 (4) (2017) 345-369. URL https://doi.org/10.1007/ s10207-016-0336-y

[4] Cisco visual networking index: Global mobile data traffic forecast update, 2016-2021 white paper, https:// www.cisco.com/c /en/us/solutions/collateral/ service-provider/visualnetworking-index-vni/mobile-white-paper-c11-520862.html, accessed: 2018-06-04.

[5] Percentage of all global web pages served to mobile phones from 2009 to 2018,https://www.statista.com/statistics/241462/global-mobile-phone-website-traffic-share accessed: 2018-07-02 
[6] E. Alepis, A. Kontogianni, C. Patsakis, Mapping the wireless coverage grid for carrier and user recommendations through crowdsourcing, in: 2017 IEEE 29th International Conference on Tools with Artificial Intelligence (ICTAI), 2017, pp. 822-829.https://doi.org/10. 1109/ICTAI.2017.00129

[7] The worlds' most valuable resource is no longer oil, but data, https://www.economist.com/leaders/2017/05/06/the-worlds-most-valuable-resource-is-nolonger-oil-but-data, accessed: 2018-06-12.

[8] A. K. Tripathy, P. K. Tripathy, N. K. Ray, S. P. Mohanty, Itour: The future of smart tourism: An iot framework for the independent mobility of tourists in smart cities, IEEE Consumer Electronics Magazine 7 (3) (2018) 32-37. doi:10.1109/ MCE.2018.2797758.URL https://doi.org/10.1109/MCE.2018.2797758

[9] J. L. Jorro-Aragoneses, M. B. D. Agudo, J. A. R. Garca, Madrid live: A context- aware recomendar system of leisure plans, in: 2017 IEEE 29th International Conference on Tools with Artificial Intelligence (ICTAI), 2017, pp. 796-801. https://doi.org/10.1109/ ICTAI.2017.00125

[10] A. Kontogianni, E. Alepis, Hotspotcrowd: A crowd sourced based application for sharing data between mobile devices, in: 2017 8th International Conference on Information, Intelligence, Systems Applications (IISA), 2017, pp. 1-6. https://doi.org/10.1109/IISA. $\underline{2017.8316438 .}$.

[11] D. V. Le, N. C. Thuong, H. Scholten, P. J. M. Havinga, Symbiotic sensing for energyintensive tasks in large-scale mobile sensing applications, in: Sensors, 2017.

[12] J. Burke, Participatory sensing, in: Proceedings of the Workshop on World- Sensor-Web (WSW 2006), Boulder, Colorado, USA, Nov., 2006.

[13] B. Guo, Z. Yu, X. Zhou, D. Zhang, From participatory sensing to mobile crowd sensing, in: Pervasive Computing and Communications Workshops (PERCOM Workshops), 2014 IEEE International Conference on, IEEE, 2014, pp. 593-598. https://doi.org/10.1109/ PerComW.2014.6815273

[14] N. D. Lane, E. Miluzzo, H. Lu, D. Peebles, T. Choudhury, A. T. Campbell, A survey of mobile phone sensing, Comm. Mag. 48 (9) (2010) 140-150. https://doi.org/10.1109/ MCOM.2010.5560598

[15] X. Sheng, J. Tang, X. Xiao, G. Xue, Sensing as a service: Challenges, solutions and future directions, IEEE Sensors journal 13 (10) (2013) 3733-3741. https://doi.org/10.1109/ JSEN.2013.2262677

[16] Z. Ou, J. Dong, S. Dong, J. Wu, A. Yl-Jski, P. Hui, R. Wang, A. W. Min, Utilize signal traces from others? a crowdsourcing perspective of energy saving in cellular data communication, IEEE Transactions on Mobile Computing 14 (1) (2015) 194-207. https://doi.org/10.1109/TMC.2014.2316517.

[17] Y. Li, J. Gao, P. P. C. Lee, L. Su, C. He, F. Yang, W. Fan, A weighted crowdsourcing approach for network quality measurement in cellular data networks, IEEE Transactions on Mobile Computing 16 (2) (2017) 300-313. https://doi.org/10.1109/TMC.2016. $\underline{2546900 .}$

[18] D. C. Brabham, K. M. Ribisl, T. R. Kirchner, J. M. Bernhardt, Crowdsourcing applications for public health, American Journal of Preventive Medicine 46 (2) (2014) 179 -187. doi: https://doi.org/10.1016/j.amepre.2013.10.016

[19] R. Montella, S. Kosta, I. Foster, Dynamo: Distributed leisure yacht-carried sensornetwork for atmosphere and marine data crowdsourcing applications, in: 2018 IEEE International Conference on Cloud Engineering (IC2E), 2018, pp. 333-339. https://doi.org/10. $\underline{\text { 1109/IC2E.2018.00064 }}$ 
[20] V. Berberidis, E. Alepis, Mycrowdcharger: Towards a crowd sourced based application for energy sharing between smartphones, 2016 7th International Conference on Information, Intelligence, Systems \& Applications (IISA) (2016) 1-4. https://doi.org/10.1109/ IISA.2016.7785382

[21] T. Yu, Z. Zhou, D. Zhang, X. Wang, Y. Liu, S. Lu, INDAPSON: an incentive data plan sharing system based on self-organizing network, in: 2014 IEEE Conference on Computer Communications, INFOCOM 2014, Toronto, Canada, April 27 - May 2, 2014, 2014, pp. 1545-1553. https://doi.org/10.1109/INFOCOM.2014.6848090

[22] Pm quit cornwall holidays over poor phone signals, https://www.bbc. com/news/ukengland-cornwall-27963341, accessed: 2018-06-12.https://powerfulsig nal.com/cell-signal-strength/

[23] T. Brandt, J. Bendler, D. Neumann, Social media analytics and value creation in urban smart tourism ecosystems, Information \& Management 54 (6) (2017) 703-713. https://doi.org/10.1016/j.im.2017.01.004

[24] P. Buonincontri, R. Micera, The experience co-creation in smart tourism destinations: a multiple case analysis of European destinations, J. of IT \& Tourism 16 (3) (2016) 285315.URLhttps://doi.org/10.1007/s40558-016-0060-5.

[25] D'Aniello, M. Gaeta, M. Z. Reformat, Collective perception in smart tourism destinations with rough sets, in: 3rd IEEE International Conference on Cybernetics, CYBCONF 2017, Exeter, United Kingdom, June 21-23, 2017, 2017, pp. 1-6. https://doi.org/10.1109/ CYBConf.2017.7985765

[26] Range of bluetooth on android, http://profandroid.com/network/Bluetooth/range-ofbluetooth.html, accessed: 2018-06-12.

[27] E. Alepis, M. Virvou, K.Kabassi: Mobile Education: Towards Affective Bi-modal Interaction for Adaptivity. iJIM 3(2): 40-45 (2009) https://doi.org/10.3991/ijim.v3i2.774

[28] Yohanes Erwin Dari, Suyoto Suyoto Suyoto, Pranowo Pranowo Pranowo:

[29] CAPTURE: A Mobile Based Indoor Positioning System using Wireless Indoor Positioning System. iJIM: 61-72 (2018) https://doi.org/10.3991/ijim.v12i1.7632

[30] Tinashe Chuchu: Technology as an Emerging Educational Tool or Tourism Information Sourcing: A Content Analysis 2006 to 2018. iJES (2019) https://doi.org/10.3991/ ijes.v7i2.10772

\section{Authors}

Aristea Kontogianni received a B.Sc in Computer Science in 2014 from Aristotle University of Thessaloniki and a MSc in Advanced Information Systems from University of Piraeus in 2017. She is currently a Ph.D. candidate in University of Piraeus that has already co-authored a number of scientific papers published in international journals. Her research interests are in the areas of Smart Cities, Smart Tourism, Mobile Software Engineering and User Modeling. Email: akontogianni@unipi.gr

Dr. Efthimios Alepis received a B.Sc. in Informatics in 2002 and a Ph.D. in 2009, both from the Department of Informatics, University of Piraeus (Greece). He is Assistant Professor in the Department of Informatics, University of Piraeus since 2013. He has authored/co-authored more than 120 scientific papers which have been published in international journals, book chapters and international conferences. Dr. Alepis is the founder of the Greek Company "Software Engineering Innovation Group - SEIG" the activities of which include, among other, production of innovative software, IT 
services and organization of international conferences. His current research interests are in the areas of Object-oriented Programming, Mobile Software Engineering, Human-Computer Interaction, Affective Computing, User Modeling and Educational Software.Email: talepis@unipi.gr

Article submitted 2019-10-03. Resubmitted 2019-12-04. Final acceptance 2019-12-08. Final version published as submitted by the authors. 